\title{
New perspectives on the role of anti-Müllerian hormone (AMH) in women
}

\author{
Renato Pasquali \\ Alma Mater Studiorum University of Bologna, Bologna, Italy \\ Correspondence to: Renato Pasquali. Via Santo Stefano 38, 40125 Bologna, Italy. Email: renato.pasquali@unibo.it. \\ Provenance: This is an invited Editorial commissioned by Section Editor Hengwei Liu, MD, PhD (Union Hospital, Tongji Medical College, \\ Huazhong University of Science and Technology, Wuhan, China). \\ Comment on: Tata B, Mimouni NEH, Barbotin AL, et al. Elevated prenatal anti-Müllerian hormone reprograms the fetus and induces polycystic \\ ovary syndrome in adulthood. Nat Med 2018;24:834-46.
}

Submitted Oct 26, 2018. Accepted for publication Nov 06, 2018.

doi: $10.21037 / \mathrm{atm} .2018 .11 .19$

View this article at: http://dx.doi.org/10.21037/atm.2018.11.19

\section{Pathophysiological and clinical aspects of anti- Müllerian hormone (AMH)}

$\mathrm{AMH}$ is a dimeric glycoprotein and a member of the transforming growth factor $\beta$ (TGF- $\beta$ ) family of growth and differentiation factors (1), and its receptors (AMHR1 and AMHR2) are located in many organs and tissues, including in the hypothalamic neurons (2). The availability of its measurement in the blood, which currently has high values of sensitivity and specificity, has considerably modified our knowledge of processes related to fertility processes, particularly in the diagnostic workup of the polycystic ovary syndrome (PCOS) (2). Its main actions are related to the development of a highly sensitive assay for measuring AMH in serum, although international reference values for the female population are not yet available. In any case, the release of AMH from the ovaries leads to an adequate quantity in the serum, proportional to the number of ovarian follicles (3). It should be emphasized there is a certain variability in AMH blood levels, depending on ovarian number of follicles and androgen levels, ethnicity, hormonal contraceptive use, and, probably, excess weight or obesity (3). Physiologically, available data support the concept that follicular AMH is a gatekeeper limiting follicle growth and estradiol (E2) production. In addition, it has been suggested that AMH may not only be a biomarker of ovarian follicle function and count in diagnosing PCOS but also in the assessment of hyperandrogenemia (4). Intriguingly, there is evidence that some important changes exist in the dynamics of circulating AMH throughout the lifespan. In fact, after a peak at birth, there is a subsequent constant reduction of AMH values at least until the early adolescent years after which, with the advent of mature age, there is a new phase of increase in AMH that persists throughout the fertile age, with a constant decline of its values in the menopause years (2). AMH can also play a role in evaluating ovarian reserve in healthy women. At present, the role of AMH in predicting fertility has been investigated in relatively few studies. Among these, prospective studies of adult women highlighted that those with low AMH had a significantly reduced chance of fecundability, which in turn was not altered in women with low-normal AMH levels $(5,6)$. Conversely, the probability of conceiving has been found to be reduced in women with high AMH levels, which suggests overt or mitigated conditions of anovulation. Undoubtedly, the true AMH values according to age may be considered to be predictive of ovarian function with increasing age (2). In any case, it should be emphasized that the ovarian reserve status throughout the lifespan requires much more long-term investigation during the age-related fertile window before menopause.

\section{AMH and its putative role in the pathophysiology of PCos}

Serum AMH is usually higher in women with PCOS compared to healthy women, and it has been suggested that it may reflect the increased number of antral follicles, where high AMH production is increased (7). Increased $\mathrm{AMH}$ blood levels have been found in the follicular fluid of women 
with PCOS (8); however, its cause is still relatively unknown, although high androgen production has been suggested as playing a potential role, since a positive correlation between androgens and AMH has been reported (9). Notably, AMH significantly decreases both the gonadotropin-induced aromatase expression in granulosa cells and the activity of the ovary-specific aromatase promoter II, which in turn favor a significant decrease in E2 production (10). The fact that $\mathrm{AMH}$ inhibits factors required for follicle growth agrees with the response of high AMH levels in PCOS. Moreover, luteinizing hormone ( $\mathrm{LH}$ ) was found to reduce AMHR2 expression in granulosa luteal cells obtained from ovulatory women with PCOS whereas it did not produce similar effects in women with anovulatory PCOS (11). Therefore, it could be suggested that levels of AMH in these ovarian follicles would be sufficient to inhibit follicle-stimulating hormone (FSH)-stimulated aromatase expression, therefore preventing the inhibitory effect of $\mathrm{E} 2$ on $\mathrm{AMH}$ production. Definitely, these data support the concept that AMH may contribute to anovulation in women with PCOS.

Recently it has been proposed that detection of $\mathrm{AMH}$ levels could be included in the diagnosis of polycystic ovarian morphology (PCOm), due to the very high significant relationship between AMH levels and follicle count on ultrasound in PCOS patients, a finding that has been specifically confirmed by the recent use of advanced technologies which make it possible to count very small follicles (1-2 mm) (12). Since evaluation of ovarian morphology, particularly follicle count, is mainly operator-dependent, the potential combination of AMH measurement with appropriate morphological evaluation by ultrasound promises to produce some benefit in the evaluation of PCOm, therefore expanding what was suggested by the Rotterdam criteria (13). Moreover, the significant correlation between androgen and $\mathrm{AMH}$ excess might support the concept that, by itself, AMH excess might be considered a biomarker of hyperandrogenemia.

\section{The role of AMH on the hypothalamic-pituitary- gonadal (HPG) axis: lessons from animal models}

AMH plays an important role in sexual differentiation and ovarian functions. On the other hand, until recently, little was known about the potential role of AMH in regulating the HPG axis and whether a dysregulation of gonadotropin secretion is associated with infertility in women, particularly in those with PCOS with various human fertility disorders, particularly PCOS. Similarly, we had limited knowledge concerning the mechanisms and factors responsible, although it has long been known that women with PCOS are characterized by high-frequency pulse and low FSH secretion (14). More recent studies have opened an important window on the potential role of $\mathrm{AMH}$ in this context. It was known that, outside the gonads, the expression of AMHR2 has also been described in the endometrium, breast, and cervix in human females, and in the developing brain and fetal lungs in mice (15). Recently, Cimino and colleagues (15) expanded these findings and reported that, based on neuroanatomical data, AMHR2 in humans is expressed in several adult brain regions including areas of the hippocampus, cortex and hypothalamus. The same authors (15) also found that AMHR2 is expressed in a significant subset of hypothalamic gonadotropin-releasing hormone (GnRH) neurons, not only in mice but also in humans. This is of importance, since a previous study had shown that approximately $12 \%$ of the GnRH-neuron population was required for pulsatile gonadotropin secretion and puberty onset, whereas slightly higher numbers of GnRH-neurons were suggested to be required for cyclical control in adult female mice (16). Using a preclinical murine model of PCOS, Cimino and co-workers (15) reported a positive correlation between AMH and LH levels, which parallels what was found in some clinical studies reporting a positive correlation between AMH and LH (but not between AMH and FSH) response dose in PCOS patients with anovulatory infertility and normal gonadotropins $(4,17)$. Undoubtedly, these findings support the hypothesis that elevated AMH blood levels might contribute to the endocrine alterations observed in PCOS, such as the significant rise in LH pulsatile secretion, which are closely related to the increased ovarian androgen production by theca cells. Interestingly, previous clinical studies $(18,19)$ had shown normal gonadotropin profiles, but significantly elevated AMH levels in pre- and peri-pubertal daughters of women with PCOS and that most of them are characterized by an early development of reproductive, hormonal and metabolic alterations, antecedents to the expression of PCOS in adult age (20). Definitely, the available data support the hypothesis that AMH may play an important role in the regulation of GnRH release, which, in turn, could be involved in the pathophysiology of infertility in women with PCOS.

\section{Elevated prenatal AMH reprograms the fetus and induces PCOS in adulthood}

In the last few years it has been clearly demonstrated 
that the pathophysiology of PCOS implies, among other things, an increase in $\mathrm{AMH}$ combined with a hypothalamic dysregulation, as most women with PCOS are characterized by elevated LH levels and altered high-frequency GnRH secretion (20). Recent evidence has also shown that exogenous $\mathrm{AMH}$ markedly increases GnRH neuron firing and $\mathrm{GnRH}$ release, at least in murine living tissue explants (15). Whether in-utero AMH may affect the development of the female fetus is relatively unknown, particularly in pregnant women with PCOS, who are characterized by high LH levels, heightened GnRH release, and high AMH. Very convincing data on the potential role of elevated prenatal $\mathrm{AMH}$ in reprogramming the fetus and favoring the development of PCOS in adulthood recently emerged from a paper by Tata and co-workers published in Nature Medicine (21). First, they confirmed that high AMH levels during pregnancy are present in PCOS women with respect to controls, therefore suggesting that excess $\mathrm{AMH}$ in utero might affect the development of the female fetus. Moreover, they reported that high AMH levels during pregnancy were higher only in normal-weight PCOS but not in those with overweight or obesity and, additionally, that $\mathrm{AMH}$ was significantly higher only in hyperandrogenic normal-weight women than in their normoandrogenic counterpart, whereas no difference was detected in hyperandrogenic obese PCOS compared to their normoandrogenic counterparts. They then moved to experimental research activities in the laboratory, using animal models. To determine whether the elevation of AMH in pregnant PCOS women is a bystander effect or a driver of the condition in the offspring, they modeled the findings observed in women with PCOS, they experimentally treated pregnant mice with $\mathrm{AMH}$, and they investigated the neuroendocrine phenotype of their female progeny postnatally. They found that AMH treatment led to an increase in the maternal neuroendocrine-driven $\mathrm{T}$ excess and reduced the placental conversion of $\mathrm{T}$ to $\mathrm{E} 2$, which resulted in a male type phenotype of the exposed female fetus and a PCOS-like phenotype in adulthood. They also reported that the affected female animals were characterized by a persistence of the activity of $\mathrm{GnRH}$ neurons and, most importantly, that treatment with cetrorelix, a GnRH antagonist, in the adult female offspring restored their neuroendocrine phenotype to a normal state. Collectively, these findings support the concept that inutero exposure to excess $\mathrm{AMH}$ may permanently alter the GnRH neuronal afferent network and its activity in the offspring and that high $\mathrm{AMH}$ may have a programming effect, leading to gestational and perinatal androgen excess and subsequent changes in the HPG axis and hormone levels of the progeny. Intriguingly, these findings appear to fit very well with previous studies demonstrating that gestational hyperandrogenism in monkeys, either occurring naturally or driven by $\mathrm{T}$ treatment, may induce a PCOSlike reproductive and metabolic trait in adulthood (22).

Overall, these findings highlight a hypothetical role for excess prenatal $\mathrm{AMH}$ exposure in the neuroendocrine dysfunctions of PCOS. Whether these findings are confirmed or not, there is no doubt that they may offer a new potential therapeutic strategy for the treatment of PCOS not only in adult women, but also in hyperandrogenic adolescent girls, who are often characterized by different phenotypes that resemble PCOS.

\section{Conclusive remarks}

The increasing interest in the pathophysiological aspects related to the role of AMH in PCOS opens new perspectives both in the definition of the various phenotypes but also in possible therapeutic developments. Until now, the diagnostic aspects have been given considerable importance, especially as regards the interpretation of ovarian morphology. In the future, it is likely that the measurement of AMH may favor a more informed understanding of the PCOS pathophysiological mechanisms whose clinical expression is often heterogeneous, which partially justifies the different diagnostic assessment of the currently available criteria (13) by gynaecologists and endocrinologists $(23,24)$.

\section{Acknowledgements}

None.

\section{Footnote}

Conflicts of Interest: The author has no conflicts of interest to declare.

\section{References}

1. Cate RL, Mattaliano RJ, Hession C, et al. Isolation of the bovine and human genes for Mullerian inhibiting substance and expression of the human gene in animal cells. Cell 1986;45:685-98.

2. Dewailly D, Andersen CY, Balen A, et al. The physiology and clinical utility of anti-Mullerian hormone in women. Hum Reprod Update 2014;20:370-85. 
3. Dewailly D, Gronier H, Poncelet E, et al. Diagnosis of polycystic ovary syndrome (PCOS): revisiting the threshold values of follicle count on ultrasound and of the serum AMH level for the definition of polycystic ovaries. Hum Reprod 2011;26:3123-9.

4. Laven JS, Mulders AG, Visser JA, et al. Anti-Mullerian hormone serum concentrations in normoovulatory and anovulatory women of reproductive age. J Clin Endocrinol Metab 2004;89:318-23.

5. Steiner AZ, Stanczyk FZ, Patel S, et al. Antimullerian hormone and obesity: insights in oral contraceptive users. Contraception 2010;81:245-8.

6. Hagen CP, Vestergaard S, Juul A, et al. Low concentration of circulating antimüllerian hormone is not predictive of reduced fecundability in young healthy women: a prospective cohort study. Fertil Steril 2012;98:1602-8.e2.

7. Park AS, Lawson MA, Chuan SS, et al. Serum anti-Mullerian hormone concentrations are elevated in oligomenorrheic girls without evidence of hyperandrogenism. J Clin Endocr Metab 2010;95:1786-92.

8. Das M, Gillott DJ, Saridogan E, et al. Anti-Mullerian hormone is increased in follicular fluid from unstimulated ovaries in women with polycystic ovary syndrome. Hum Reprod 2008;23:2122-6.

9. Carlsen SM, Vanky E, Fleming R. Anti-Mullerian hormone concentrations in androgen-suppressed women with polycystic ovary syndrome. Hum Reprod 2009;24:1732-8.

10. Pellatt L, Rice S, Mason HD. Anti-Mullerian hormone and polycystic ovary syndrome: a mountain too high? Reproduction 2010;139:825-33.

11. Pierre A, Peigne M, Grynberg M, et al. Loss of LHinduced down-regulation of anti-Mullerian hormone receptor expression may contribute to anovulation in women with polycystic ovary syndrome. Hum Reprod 2013;28:762-9.

12. Fraissinet A, Robin G, Pigny P, et al. Use of the serum anti-Müllerian hormone assay as a surrogate for polycystic ovarian morphology: impact on diagnosis and phenotypic classification of polycystic ovary syndrome. Hum Reprod 2017;32:1716-22.

13. The Rotterdam ESHRE/ASRM-Sponsored PCOS Consensus Workshop Group 2004. Revised consensus on diagnostic criteria and long-term health risks related to polycystic ovary syndrome (PCOS). Hum Reprod 2004;19:41-7.

14. Taylor AE, McCourt B, Martin KA, et al. Determinants of abnormal gonadotropin secretion in clinically defined women with polycystic ovary syndrome. J Clin Endocrinol Metab 1997;82:2248-56.

15. Cimino I, Casoni F, Liu X, et al. Novel role for antiMüllerian hormone in the regulation of $\mathrm{GnRH}$ neuron excitability and hormone secretion. Nat Commun 2016;7:10055.

16. Herbison AE, Porteous R, Pape JR, et al. Gonadotropinreleasing hormone neuron requirements for puberty, ovulation, and fertility. Endocrinology 2008;149:597-604.

17. Catteau-Jonard S, Pigny P, Reyss AC, et al. Changes in serum anti-mullerian hormone level during low-dose recombinant follicular-stimulating hormone therapy for anovulation in polycystic ovary syndrome. J Clin Endocrinol Metab 2007;92:4138-43.

18. Sir-Petermann T, Codner E, Maliqueo M, et al. Increased anti-Mullerian hormone serumconcentrations in prepubertal daughters of women with polycystic ovary syndrome. J Clin Endocrinol Metab 2006:91:3105-9.

19. Crisosto N, Codner E, Maliqueo M, et al. Anti-Mullerian hormone levels in peripubertal daughters of women with polycystic ovary syndrome. J Clin Endocrinol Metab 2007;92:2739-43.

20. Goodarzi MO, Dumesic DA, Chazenbalk G, et al. Polycystic ovary syndrome: etiology, pathogenesis and diagnosis. Nat Rev Endocrinol 2011;7:219-31.

21. Tata B, Mimouni NEH, Barbotin AL, et al. Elevated prenatal anti-Müllerian hormone reprograms the fetus and induces polycystic ovary syndrome in adulthood. Nat Med 2018;24:834-46.

22. Abbott DH, Nicol LE, Levine JE, et al. Nonhuman primate models of polycystic ovary syndrome. Mol Cell Endocrinol 2013;373:21-8.

23. Cussons AJ, Stuckey BG, Walsh JP, et al. Polycystic ovarian syndrome: marked differences between endocrinologists and gynaecologists in diagnosis and management. Clin Endocrinol (Oxf) 2005;62:289-95.

24. Conway G, Dewailly D, Diamanti-Kandarakis E, et al. European survey of diagnosis and management of the polycystic ovary syndrome: results of the ESE PCOS Special Interest Group's Questionnaire. Eur J Endocrinol 2014;171:489-98.

Cite this article as: Pasquali $\mathrm{R}$. New perspectives on the role of anti-Müllerian hormone (AMH) in women. Ann Transl Med 2018;6(Suppl 2):S94. doi: 10.21037/atm.2018.11.19 